\title{
Téoros
}

Revue de recherche en tourisme

\section{Prospective touristique : essai méthodologique}

\section{Bernard Morucci}

Volume 12, numéro 2, juillet 1993

La prévision-prospective du tourisme

URI : https://id.erudit.org/iderudit/1078020ar

DOI : https://doi.org/10.7202/1078020ar

Aller au sommaire du numéro

Éditeur(s)

Université du Québec à Montréal

ISSN

0712-8657 (imprimé)

1923-2705 (numérique)

Découvrir la revue

Citer cet article

Morucci, B. (1993). Prospective touristique : essai méthodologique. Téoros, 12(2), 10-17. https://doi.org/10.7202/1078020ar d'utilisation que vous pouvez consulter en ligne.

https://apropos.erudit.org/fr/usagers/politique-dutilisation/ 


\title{
Prospective touristique: essai méthodologique
}

\author{
Bernard Morucci"
}

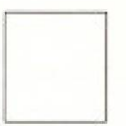

Dans un monde caractérisé par la montée des incertitudes et par le risque de ruptures de tendances, hier à long terme mais de plus en plus à moyen et courtterme, la prise en compte de l'avenir est devenue indispensable pour éclairer l'action du présent. Il ne faut pas attendre l'urgence, les crises ou les catastrophes pour agir mais plutôt adopter une démarche préventive destinée à anticiper les problèmes futurs. Les actions à engager pour les résoudre seront diffếrentes selon ce que sera l'avenir et devront être adaptées aux formes qu'il prendra. Ainsi, à l'inverse de la prévision classique dans laquelle le futur se présente suivant une ligne unique et prédéterminée dans le prolongement du passé, la démarche prospective aide à la compréhension d'un monde incertain, complexe, en constante mutation, pour lequel l'avenir n'est plus tracé mais multiple, indéterminé et constituédeplusieurs futurs possibles. Elle s'appuie pour cela sur une vision globale des phénomènes ếtudiés, sur le caractère évolutif de leurs structures et sur les relations dynamiques entre les différentes variables qui les représentent (cf. encadré $\mathrm{n}^{\circ}$ 1).

La prospective s'est développée à la fin des années 1960 pour tenir compte à la fois des erreurs de plus en plus nombreuses de la prévision et de la complexité croissante des phénomènes étudiés. Dans le domaine du tourisme, en Europe, elle est apparue beaucoup plus tard car les administrations et les professionnels de ce secteur ont longtemps défini leurpolitique ou leur stratégie sur la base de prévisions de flux de visiteurs supposant un environnement stable; depuis les années cinquante, ils ont raisonné sans tenir compte des changements dans le monde etde leur accélération: doublement de la population mondiale en quarante ans, transformation des mentalités et modification des comportements de la demande touristique et des structures de la société, effets des progrès technologiques sur la

Monsieur Bernard Morucci est professeur et responsable de la formation doctorale en tourisme international à l"Universiló Rene Descartes et au Conservatoire National des Arts et Métiers.

\begin{tabular}{|c|c|c|}
\hline \multicolumn{3}{|c|}{$\begin{array}{l}\text { ENCADRE No } 1 \\
\text { Tableau comparatif de la prévision classique } \\
\text { et de la prospective }\end{array}$} \\
\hline & $\begin{array}{l}\text { Prévision } \\
\text { classique }\end{array}$ & Prospective \\
\hline Vision & $\begin{array}{l}\text { Parcellaire } \\
\text { «toutes choses égales } \\
\text { par ailleurs* }\end{array}$ & $\begin{array}{l}\text { Globale } \\
\text { «rien n'est égal par } \\
\text { ailleurs }\end{array}$ \\
\hline Variables & $\begin{array}{l}\text { Quantitatives, } \\
\text { objectives et connues }\end{array}$ & $\begin{array}{l}\text { Qualitatives, } \\
\text { quantifiables ou non } \\
\text { subjectives, connues ou cachées }\end{array}$ \\
\hline Relations & $\begin{array}{l}\text { Statiques, structures } \\
\text { constantes }\end{array}$ & $\begin{array}{l}\text { Dynamiques, } \\
\text { structures évolutives }\end{array}$ \\
\hline Explications & Le passé explique l'avenir & $\begin{array}{l}\text { L'avenir est la raison d'être du } \\
\text { présent }\end{array}$ \\
\hline Avenir & Unique et certain & Multiple et incertain \\
\hline Méthode & $\begin{array}{l}\text { Modèles déterninistes } \\
\text { et quantitatifs } \\
\text { (économétriques) } \\
\text { (mathématiques) }\end{array}$ & $\begin{array}{l}\text { Analyse intentionnelle } \\
\text { Modeles qualitatifs } \\
\text { (analyse structurelle) } \\
\text { et stochastiques } \\
\text { (impacts croisés) }\end{array}$ \\
\hline Attitude face à l'avenir & $\begin{array}{l}\text { Passive ou adaptative } \\
\text { (avenir subi) }\end{array}$ & $\begin{array}{l}\text { Active et creative } \\
\text { (avenir woulu) }\end{array}$ \\
\hline
\end{tabular}

Source: Michel Godet, Crise de la prévision, essor de la prospective, PUF, 1977.

communication, la distribution et le transport touristiques, etc. Tout au plus observait-on quelquesvariantes par rapport à un scénario tendanciel unique obtenu par extrapolation de données antérieures et postulant que *toutes choses demeuraient égales par ailleurs\%. Il faut attendre la fin des années soixante-dix pour prendre en compte les phénomènes qualitatifs, de discontinuité et de rupture parallèlement à une prise de conscience de l'importance de l'environnement et à la nouvelle conception du tourisme telle que l'a définie la Déclaration de Manille (cf. encadré $\mathrm{n}^{\circ} 2$ ).

Les premières études prospectives significatives datent de cette époque. La plupart d'entre elles se ramènent à la construction de scénarios déterminés à partir d'une approche «littéraires, c'est-à-dire en utilisant une méthode qui fait appel uniquement à la réflexion et au raisonnement, à l'exclusion de tout instrument mathématique ou informatique. D'autres reposent sur des méthodes deconsultation d'experts ${ }^{(1)}$ telles que la méthode Delphi fondée sur l'utilisaton systématique d'un jugement intuitif d'un groupe d'experts; ceux-ci sont interrogés à l'aide de questionnaires successifs afin demettreen évidencedes convergences d'opinions et de dégager d'éventuels consensus. En 1978, Jost Krippendorf a, pour la première fois, utilisé cette technique au niveau d'un pays ${ }^{(2)}$; depuis, des enquêtes Delphi réalisées dans le secteur du tourisme font l'objet de nombreuses publications. Les études prospectives qui se sont appuyées sur une succession d'outils et de méthodes scientifiques et informatisées sont par contre beaucoup plus rares, même encore aujourd'hui ${ }^{(3)}$. C'est l'une des premières d'entre elles, «Projet 
d'optimisation économique du domaine skiable de Trois Vallées»(4) qui servira d'illustration et de support à la présentation des différentes phases méthodologiques de la prospective touristique (cf. encadré $\mathrm{n}^{\circ} 3$ ).

Composé des vallées de Saint-Bon, des Allues et des Belleville, le domaine skiable des Trois Vallées (cf. carte), au coeur des Alpes françaises, est un des plus vastes du monde. Son aménagement en 1979 - date de l'étude - était:

- quasi terminé dans la vallée de SaintBon;

- en cours d'achèvement dans la vallée des Allues, la station du Mottaret restant seule à développer;

- à réaliser dans la vallée des Belleville, notamment les stations des $M$ Menuires et de Val Thorens.

Deux options de développement étaient possibles:

- prolonger en les coordonnant les actons déjà engagées, en supposant que le «marché de la neige» se développe dans l'avenir de la même façon que dans le passé: c'est l'approche prévisionnelle;

- élaborer plusieurs scénarios d'évolution de la demande de sports d'hiver à l'horizon 1995, ce qui implique d'intégrer à la fois l'évolution de l'environnement économique et celle des mutations possibles dans le mode de consommation de loisirs: c'est la démarche prospective qui, par son approchedans un cadre deréflexion plus global, permet de proposer des politiques correspondant aux différents scénarios et d'en mesurer les conséquences en termes d'investissement et de retombées socio-économiques (chiffre d'affaires, emploi, etc.).

Pour donner du problèmeétudié uneimage aussicomplèteque possible, il estcommode de recourir à l'analyse de système *qui consisteàmettre en valeur le fait quel'objet examiné doit être placé dans un contexte plus large que son système original, le terme d'analyse servantà souligner qu'il est utile de décomposer les problèmes complexes en éléments constituantsw(5). La délimitation dusystème des \& Trois Vallées

ENCADRÉ No 2

Caractéristiques du tourisme avant et après Manille

Avant Manille

- Tourisme international spontané (au maximum: planification sectorielle)

- Stratégie des entreprises

- Aspects économiques privilégiés

- Aspects matériels dominants

- Profit maximisé

- Quantitatif

- Orienté sur les prix

- Croissance

- Vacances (comment passer les vacances)

- Besoin de compensation, évasion

- Passif

- Guides

- Produits stéréotypés (voyages à

forfait basés sur les 4S)

- Propagande-réclame

- Personnel recruté dans d'autres sphères

- Touriste manipulé

- Ignorance de langues étrangères

- Détérioration de l'environnement

- Enclaves et ghettos touristiques

- Saturation

- Utilisation des régions les plus précieuses

- Automobile

- Arrivées

\section{Après Manille}

- Tourisme global planifié (planification intégrée)

- Politique touristique au niveau national

- Importance complexe (politique, sociale, économique, culturelle, éducative, protection de l'environnement, qualité de la vie)

- Les aspects non matériels dominent

- Apports économiques optimaux + avantages non matériels

- Qualitatif

- Accent mis sur la valeur

- Développement

- Déplacements de vacances, de fin de semaine et d'une journée (comment vivre)

- Besoin de diversité se manifestant par les motivations variées et un tourisme de plus en plus actif

- Actif

- Animateurs

- Produits différenciés (voyages individuels autodépendants)

- Informations et éducation

- Personnel spécialisé

- Touriste émancipé

- Connaissance des langues de vocation universelle, telles que l'espéranto

- Protection de l'environnement

- Intégration à la population locale

- Capacité de charge de la région, considérée à l'avance

- Formation de réserves, conservation de l'agriculture

- Transports publics

- Durée de séfour

Source: OMT, Madrid, 1980.

faite à partir d'interviews, de brainstormings, de réunions de réflexion collective et d'entretiens non directifs a retenu soixante-douze variables (cf. encadré $n^{\circ} 4$ ): quarante, internes, sont caractéristiques du domaine skiable lui-même et trente-deux, externes, de son environnement. Une analyse plus fine de ces deux sous-systèmes débouche sur des regroupements homogènes pour chacun d'eux: sports-loisirs, hébergement, vie locale, sociologie, économie et commune d'une part; département, État, consommateurs et environnement international d'autre part. L'examen des relations entre toutes cesvariables aideà la compréhension et explique la dynamique du système. II s'effectue grâce à la matrice d'analyse structurelle $e^{(6)}$ qui permet de mesurer les influences directes de chaque variable sur toutes les autres et de sélectionner les principales d'entre elles ( $c f$, encadré $n^{\circ} 5$ ). L'examen systématique des relations indi- rectes entre toutes les variables, effectué par la méthode Mic $\mathrm{Mac}^{(0)}$, améliore la connaissance du système. Il a confirmé, en les amplifiant parfois, les principaux résultats obtenus par la méthode directe mais a surtout mis l'accent sur l'influence motrice indirecte de 4 autres variables initialement écartées del'analyse par suitedeleurs effets directs limités:

- marché de l'immobilier (58);

- pression écologique (59);

- développement du ski de fond (60);

- et du ski de randonnée (61).

La figure $\mathrm{n}^{\circ} 1$ donne une représentation des 21 variables essentielles hiérarchisées en fonction de leurs indices de motricité et de dépendance.

Portant sur les quatre groupes de variables essentielles, l'analyse explicative du système des *Trois Vallées» comporte: 


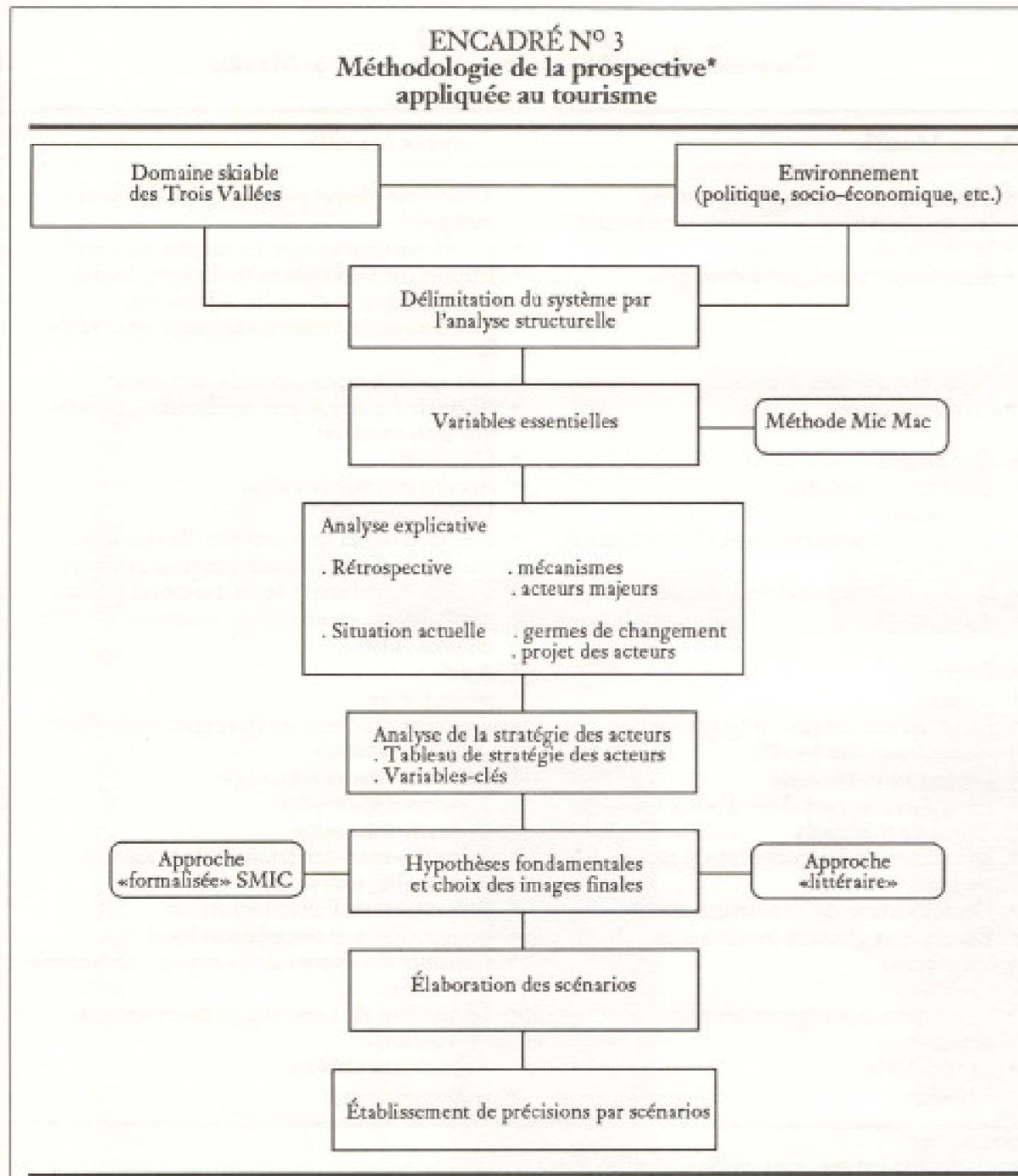

*Cf.: Michel Godet, De l'anticipation à l'action, Dunod, 1991: La méthode des scénarios.

\section{FIGURE $N^{\circ} 1$}

Indice de motricité

I Variables de commande

- Politique nationale

- Politique départementale

- Promotion

- Immobilier (prix, banalisation)

- Niveau de vie - loisirs

- Autres activités

- Domaine

- Acces

- Ecologie

IV Variables peu sensibles

- Résultats des sociétés de remontées mécaniques et d'aménagement

- Emploi dans le reste du département

- Fréquentation des autres lieux
II Variables instables

- Nombre de lits

- Commerce

- Budgets départementaux

- Budgets communaux

III Variables sensibles

- Fréquentation étrangère

- Emploi dans les 3 vallées

- Artisanat

- Aspects sociaux

- Démographie dans les 3 vallées

Indice de dépendance

- une étude rétrospective destinée à êliminer les facteurs conjoncturels et à dégager les mécanismes et les acteurs les plus déterminants; ainsi, se trouvent mis en évidence le rôle moteur que jouait dans la France de 1978 le pouvoir politique centralisé $(43,44$ et 48), l'importance des procédures foncières (12 et 58$)$ et des contraintes budgétaires (36et 45) ainsi quele frein des facteurs socio-économiques locaux $(24,25$ et 26$)$;

- une étude de la situation actuelle qui permet de repérer les germes de changement: dès 1978, la méthode Mic Macrévélaitl'importance devariables caractéristiques de la demande de la fin des années 1980: écologie et développement de nouvelles formes de loisirs sportifs privilégiant la nature et le comportement à la fois plus individualiste et plus actif du touriste. Cette phase comprend également le recensement, à partir de sources documentaires, des projets des neuf principaux acteurs impliqués et dégagés par l'étude rértrospective: État et Préfet/Conseils général et munici$\mathrm{pal} /$ Administration/Population locale/Grand public/Promoteurs immobiliers/Sociétés de remontées mécaniques et aménageurs privés et publics. Ce sont eux qui vont aider à piloter le système. A titre d'exemple, l'encadré $n^{\circ} 6$ présente les points principaux du projet de développement de l'acteur *Conseil généralm.

La dynamique du système rapporté à ses variables essentielles s'effectue en confrontant les moyens d'action des différents acteurs une fois leurs projets recensés (cf. encadré $n^{\circ} 7$ ). La première étape consiste donc à collecter le maximum d'information sur la stratégie de chacun. Cetteopếration délicate s"effectue surtout à partir d'interviews auprès des différents protagonistes. L'ensemble de l'information (projets et moyens d'action) estensuiterassemblé dans le *tableau de stratégie des acteurs $*{ }^{(9)}$ dont l'étude détaillée, case par case, permet de repérer lespossibilitésd'allianceetdeconflit et de les regrouper autour d'enjeux stratégiques tels que:

- l'intégration du développement touristique dans le département;

- la répartition budgétaire; 
ENCADRÉ No 4

Liste des variables retenues

Variables internes

\begin{tabular}{|c|c|c|}
\hline & 1. & $\begin{array}{l}\text { Dimension du domaine équipé } \\
\text { Surface des pistes }\end{array}$ \\
\hline Sport & & Charge des pistes (nombre de skicurs/hect.) \\
\hline \multirow[t]{7}{*}{ Loisir } & & Capacité des remontées \\
\hline & & Qualité des pistes \\
\hline & & Animation \\
\hline & & Pourcentage de non skieurs \\
\hline & 8.1 & Paysage - environnement \\
\hline & 9. & Nombre de lits \\
\hline & 10. & $\begin{array}{l}\text { Pourcentage de lits banalises } \\
\text { Nombre de lits ruraux }\end{array}$ \\
\hline \multirow{7}{*}{$\begin{array}{l}\text { Héber- } \\
\text { gement }\end{array}$} & & $\begin{array}{l}\text { Nombre de lits ruraux } \\
\text { Coût foncier }\end{array}$ \\
\hline & 13. & Coût à la construction \\
\hline & & Rapport séjour/week-end \\
\hline & 15. 1 & Fréquentation estivale \\
\hline & 16. 1 & Prix de vente de l'immobilier \\
\hline & 17. & Accès route, fer, air \\
\hline & 18. ( & Commerce \\
\hline \multirow{6}{*}{$\begin{array}{l}\text { Vie } \\
\text { locale }\end{array}$} & 19. 1 & Effort de promotion \\
\hline & 20. & Artisanat \\
\hline & $21+$ & Agriculture \\
\hline & 22. & $\begin{array}{l}\text { Coût de la ressource en eau } \\
\text { Imare de marque (snobisme de la station) }\end{array}$ \\
\hline & 24. & Démographie dans les 3 vallées \\
\hline & 25.1 & Réactions sociologiques des habitants \\
\hline \multirow[t]{7}{*}{ Sociologie } & 26. & Climat social \\
\hline & 27.1 & Emploi total dans les 3 vallées \\
\hline & 28. 1 & Emplois savoyards \\
\hline & 29. 1 & Emplois permanents \\
\hline & 30. & Composition sociologique des séjournants \\
\hline & & Politique tarifaire des remontées \\
\hline & 32. & Politique salariale des remontées \\
\hline & 35. & $\begin{array}{l}\text { Resultats des sociétés d'exploitation } \\
\text { Résultats des sociétés d'aménagement }\end{array}$ \\
\hline & 35.1 & Résultats de la SCTVABEL \\
\hline \multirow{4}{*}{ Commune } & 36. & Intérüts loconx narticuliens \\
\hline & & $\begin{array}{l}\text { Appartenance politique du Maire/Conseil } \\
\text { Général }\end{array}$ \\
\hline & 39. & Satisfaction des électeurs \\
\hline & 40,1 & Inscriptions sur les listes électorales \\
\hline
\end{tabular}

\section{Variables externes}

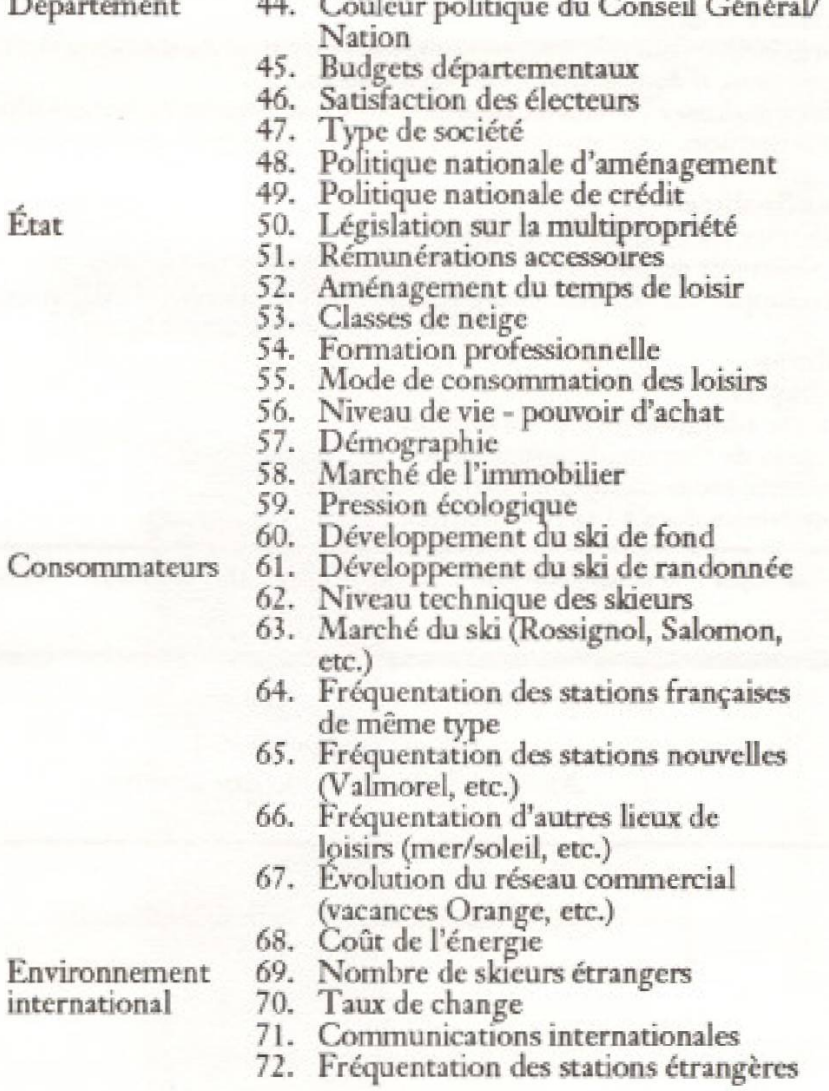

41. Emplois induits dans le reste du département par les 3 Vallées

42. Emplois dans le département (hors 3 Vallées)

43. Couleur politique du Conseil Général

4. Couleur politique du Conseil Général/

47. Type de société

48. Politique nationale d'aménagement

Politique nationale de credit

51. Rémunérations accessoires

2. Amenagement du temps de loisir

5. Mode de consommation des loisirs

56. Niveau de vie - pouvoir d'achat

59. Pression écologique

2. Niveau technique des skieurs

3. Marché du ski (Rossignol, Salomon,

de même type

des stations nouvelles (Valmorel, etc.)

loisirs (mer/soleil, etc.)

72. Fréquentation des stations étrangères

ENCADRÉ No 5

Classement direct des principales variables

$\begin{array}{ll}\begin{array}{l}\text { Variables externes } \\ \text { motrices }\end{array} & \begin{array}{l}\text { Variables internes } \\ \text { dépendantes }\end{array}\end{array}$

Politique nationale d'aménagement (48)

Budgets communaux (36)

Niveau de vie/pouvoir d'achat (56)

Réaction sociologique des habitants (25)

Type de socićté (47)

Commerce (18)

Couleur politique du Conscil général (43)

Nombre de lits (9)

Emploi total *Trois Vallées* (27) 
ENCADRE No 6

Projet du Conseil général de la Savoie

\section{Objectifs}

- Assurer une priorité aux habitants du département dans la mise en valeur de la montagne: - en favorisant l'accès aux emplois nouveaux (reconversion, formation aux métiers de la neige et du tourisme);

- en améliorant la qualité des emplois proposés (politique salariale, stabilité des emplois avec le développement de nouvelles formes de tourisme).

- Encourager la jeunesse du département à fréquenter et aimer la montagne à l'aide de mesures destinées à favoriser la clientèle de ski à la journée et l'accès de toutes les classes sociales à la neige.

- Faire profiter toutes les communes du département du tourisme wor blanc* en ternes de promotion, d'équipements, de voies d'accès.

- Mieux maitriser l'avenir et assurer une bonne gestion en termes d'investissement et de redistribution.

Missions finalités

- Défendre les intérêts du département.

- Se maintenir au pouvoir.

- Développer des équipements collectifs dans l'ensemble du département.

\section{Contraintes}

- Politiques,

- Tutelle administrative et financière.

- Gestion de l'espace départemental.

- Contexte socio-culturel du département.

- Contraintes dues à l'environnement (climat, ressources).

Source: Projet d'optimisation économique du domaine skiable des *Trois Valléess.
- le maintien du milieu naturel (notamment dans son rapport à l'agriculture et à l'environnement);

- l'emploi;

- les investissements.

Pour chacun de ces enjeux, on précise les intérêts convergents et antagonistes ainsi que les moyens d'action des acteurs concernés. Un graphe de synthèse (la figure n ${ }^{\circ}$ 2 en donne un extrait), visualise l'ensemble de ces informations en tenant compte du fait que certains acteurs peuvent être en accord sur certains enjeux et en opposition sur d'autres. Il souligne l'importançe et la force de la puissance publique (Etat et Préfet) qui s'exerce à travers les délégations départementales à l'équipement(DDE) et à l'agriculture (DDA) ainsi que, pour les aspects techniques plus directement liés au tourisme, à travers le Service d'études et d'aménagement touristiques de la montagne (SEATM). Le pouvoir des acteurs professionnels et des populations se situe davantage sur le plan de la mobilisation politique et au niveau des mouvements d'opinions et du chantage à l'implantation et à l'emploi.

Le graphe de synthèse permet donc de comprendre le jeu stratégique d'ensemble dont l'intérêt est de dégager les questionsclés pour l'avenir ( $c f$. encadré $\mathrm{n}^{\circ} 8$ ). Cellesci sont soumises à des critères ou à des facteurs exogènes dont la projection dans le futur est incertaine - par exemple l'issue de conflits possibles -; il est alors nécessaire de formuler pour chacune d'elles des hypothèses que, dans la pratique, on évite de multiplier pour ne pas alourdir l'étude. Après confrontation des variables-enjeux et des rapports de force entre acteurs, sept hypothèses ont été retenues pour servir de base à la construction des scénarios.

A1- Relance des stations de sports d'hiver axée sur la clientèle internationale.

A2- Transfert sur les collectivités locales du développement des stations, accompagné, du point de vue économique, d'un frein aux investissements immobiliers et à la consommation d'énergie avec, du point de vue politique, un discours écologique.

B1- Engagement du département dans l'aménagement, ce qui implique la relancedessociétésd'aménagement, le rachat des remontées mécaniques

Source: Michel Godet, Prospective et planification stratégique, Économica, 1985. 

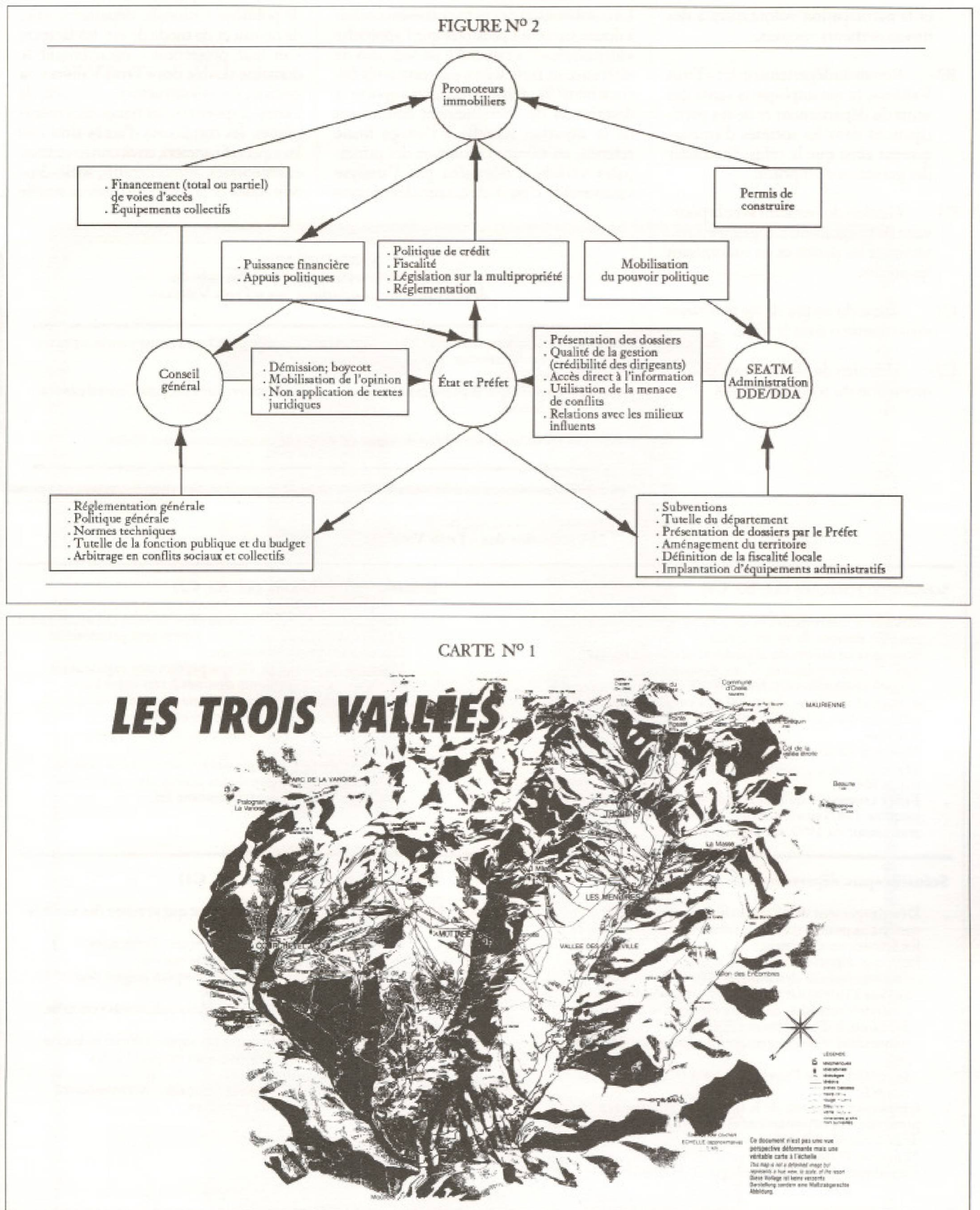

15 TEOROS - Vol. 12, no 2, juilet 1993 
et la participation volontariste à des investissements routiers.

B2- Retraitdudépartementdes *Trois Vallées\%, ce qui implique la vente des actifs du département et de ses participations dans les sociétés d'aménagement ainsi que le refus d'accorder des garanties d'emprunt.

B3- Gestion del'existant avec la poursuite de programmes engagés en minimisant les pertes et en maximisant les profits.

C1- Baisse du niveau de vie en France mais maintien dans la $\mathrm{CEE}$.

C2- Maintien du niveau de vie et augmentation du temps de loisirs.
La combinaison de ces hypothèses conduit à douze scénarios possibles que l'approche *formalisées (9) a ramené à un scénario de référence et trois scénarios contrastés (cf. encadré $n^{\circ}$ 9). Chacun d'eux comporte la description du cheminement conduisant de la situation actuelle à l'image finale retenue, en suivant l'évolution des principales variables dégagées par l'analyse structurelle: c'est-à-dire, outre les options de politique nationale, départementale, de niveau et de mode de vie, les facteurs - et leur projection - caractérisant le domaine skiable des * Trois Valléesw, sa gestion, ses infrastructures d'accueil, de loisirs et sportives, les remontées mécaniques, les conditions d'accès ainsi que lesaspectsfinanciers, environnementaux, énergétiques, administratifs, socio-économiques et la demande prévisionnelle

\section{ENCADRÉ No 8 \\ Quelques questions-clés pour l'avenir du développement touristique des * Trois Vallées: \\ A- La politique nationale d'aménagement (imposée par l'Êtat et mise en place par les administrations). \\ B- La politique départementale d'aménagement qui est du ressort du Conseil général de Savoie. \\ C- L'évolution du niveau de vie et du mode de consommation des loisirs.}

ENCADRÉ No 9

Les scénarios des * Trois Vallées*

\section{Scénario de référence $(\mathrm{A} 2, \mathrm{~B} 3, \mathrm{C} 1)$}

- Absence d'intervention et de politique spécifique de l'État pour les stations de sports d'hiver.

- Statu quo au niveau du département qui se contente, par suite de moyens limités, d'une gestion saine.

- Actions communes tendant à développer la synergie des stations et du Conseil général et à emporter leur adhésion dans le but de renforcer l'image des « Trois Vallées:

- Maintien du niveau de vie en Europe. Aspiration des Français dont le pouvoir d'achat diminue, a plus de temps libre.

- Faible croissance de la demande française et étrangère jusqu'en 1987 , suivie à terme d'une légère décroissance avec retour en 1995 aux chiffres de 1978 .
Scénario *privatisation* $(\mathrm{A} 2, \mathrm{~B} 2, \mathrm{C} 2)$

. Désengagement de l'Êtat et du département qui soutiennent d'autres activités économiques jugées plus prioritaires. Pression environnementaliste.

- Maintien du niveau de vie qui permet une exploitation privée des aménagements destinés à répondre à une demande importante (la plus forte des quatre scénarios) aussi bien française qu'étrangère et nécessitant les aides les plus faibles de l'extérieur pour le développement des stations.

- Nécessité d'une forte agressivité commerciale pour la vente du grand nombre de lits prévus ainsi que d'une animation de qualité, hiver comme été.
Scénario *parc départemental $(\mathrm{A2}, \mathrm{B} 1, \mathrm{C} 2)$

Désengagement de l'État mais réglementations nationales pour favoriser la protection de la nature et les économies d'énergie.

- Politique départementale:

- d'investissement dynamique, d'intégration locale, de soutien a l'artisanat traditionnel, de promotion d'activités estivales, de ski de randonnée et de fond, de maintien d'activités rurales;

- volontariste d'aménagement (limitation du nombre de lits);

- de protection de l'environnement (reforestation, parc naturel).

Croissance soutenue de la demande française (en particulier du département et de la région) mais modérée de la demande étrangère.

Maintien du niveau de vie en France et en Europe.

Importance accrue de l'écologie et de la qualité de la vie.

\section{Scénario *exportation* (A1, B2, C1)}

- Désengagement du département qui se retire des sociétés d'aménagement.

- Baisse du niveau de vie des Français. Difficultés sectorielles. Chồmage en augmentation.

- Relance par l'Etat d'un nouvesu eplan neige* destiné a: - attirer les touristes européens;

- favoriser la conversion d'emplois industriels vers ceux du secteur tertiaire;

- développer les aménagements support d'une industrie touristique «piêge à devises au moyen d'aides diverses et de subventions.

- Stabilisation de la demande française. Accroissement sensible de la demande étrangère. 


\begin{tabular}{|c|c|c|c|c|}
\hline \multicolumn{5}{|c|}{$\begin{array}{l}\text { ENCADRÉ No } 10 \\
\text { Prévisions des principaux indicateurs de } \\
\text { développement touristique }\end{array}$} \\
\hline \multirow[b]{2}{*}{ Indicateurs } & \multicolumn{3}{|c|}{ Scénarios } & \multirow[b]{2}{*}{$\begin{array}{l}\text { *privati- } \\
\text { sation* }\end{array}$} \\
\hline & $\begin{array}{c}\text { de } \\
\text { référence }\end{array}$ & $\begin{array}{l}\text { *pare } \\
\text { départe- } \\
\text { mental. }\end{array}$ & $\begin{array}{l}\text { wexpor- } \\
\text { tation* }\end{array}$ & \\
\hline $\begin{array}{l}\text { Nombre de lits-touristes à créer } \\
\text { Chiffre d'affaires total induit }{ }^{*} \\
\text { Chiffre d'affaires induit en devises* } \\
\text { T.V.A." } \\
\text { Emplois totaux créés } \\
\text { dont } 1 / 3 \text { permanents }\end{array}$ & $\begin{array}{r}13000 \\
240 \\
60 \\
24 \\
1500 \\
450\end{array}$ & $\begin{array}{r}22000 \\
375 \\
140 \\
37,5 \\
2700 \\
750\end{array}$ & $\begin{array}{r}45000 \\
765 \\
630 \\
76,5 \\
4500 \\
1350\end{array}$ & $\begin{array}{r}50000 \\
850 \\
380 \\
85 \\
5000 \\
1500\end{array}$ \\
\hline Exprimé en millions de fra & ancs. & & & \\
\hline
\end{tabular}

Source: Projet d'optimisation économique du domaine skiable des Trois Vallées.

de sports d'hiver. Les images finales obtenues sont assorties de prévisions chiffrées destinées à éclairer la prise de décision des acteurs impliqués (cf, le tableau de l'encadré $n^{\circ} 10$ ). Les valeurs des principaux indicateurs, observées au début des années 1990 , se situent dans une fourchette délimitée par les prévisions des scénarios «de références et «parc départemental»s. En fait, le projet d'optimisation économique du domaine skiable des $*$ Trois Vallées» était plutôt destiné à présenter un cadre de réflexion pour aider les décideurs à élaborer des objectifs de développement touristique à long terme quà être un outil stratégique. En ce sens, il se situe dans l'esprit des principales études deprospective effectuées dans les domaines du tourisme et de l'environnementjusqu'au débutdes années 1980. Qu'aujourd'hui de nouvelles méthodes et outils scientifiques informatisés ${ }^{(10)}$ conferent à la prospective davantage de rigueur, que son association avec la planification stratégique ${ }^{(11)}$ la rende plus opérationnelle, voilà qui ne peut que renforcer sa crédibilité et accroître son utilisation.

C'est pourquoi, elle apparaît maintenant commeun instrument incontournablepour tous ceux qui éprouvent, dans le domaine du tourisme notamment, un besoin croissant d'éclairage à long terme. Les acteurs decesecteurvont devoir systématiquement entreprendre une démarche prospective car toute décision à longue portée repose de plus en plus sur une vision du futur. Parce que, aussi, toute politique touristique en cours de développement peut, à terme, entraîner des conséquences (qu'il faut prévoir par la veille prospective) contraires aux objectifs visés. Enfin, parce que la prospective fournit les moyens concrets de juger si, face à un problème de décision qui engage un aménagement touristique, *une solution préférable dans une optique à court ou moyen terme continue à l'être sí on la replace dans une perspective beaucoup plus lointaines ${ }^{(12)}$. Au-delà de ces trois raisons fondamentales, la pratique de la prospective touristique ne peut que se développer dans un secteur socio-économique appelé à devenir le plus important en l'an 2000 car elleest une opportunité pour:

- sedoter d'un outil de réflexion et d'un cadre méthodologique cohérent et rigoureux en vue d'anticiper les changements pour mieux s'y adapter et dépasser les facteurs d'inertie (habitudes, mentalités, comportements, rigidités, etc.);

- répondre à la nécessité d'y voir plus clair en réduisant les incertitudes face aux solutions technologiques à venir, à l'irruption de nouveaux acteurs, aux nouvelles potentialités du marché, à la concurrence internationale et aux mutations socio-culturelles.

Pratiquée ainsi dans le domaine du tourisme, la prospective ne peut que contribuer efficacement à la mise en oeuvre d'un développement durable.
Notes et réferences

(1) Fabrice Roubelat, Le proepective, mbthodes et outile, dans oe meame numbro.

(2) Jost Krippendort, Touriamus in jahre 2010: eine Delphi-Umfrage ubber die xukuenftige Entwicklung des Touriamus in der Schwek. Universitat Bern, Fobruar 1980.

(3) Voir en particulier deux études effectuées pour le Ministere français de l'Environnement et du Cadre de Vie, Les determinante de la demande de biens d environnement, par Rerry Barro (GERPA) et Michel Godet (SEMA Prospective), Paris, 1978; et Eléments pour une proepectlve environnement-tourlame: vers une stuatígie a longterme de gestlon de renvironnement ot de rese rapports avec le touriame, parle GERPA, Paris, 1960.

(4) Ce projet a été réalisés en 1979 par Jean-Louis Blanot, Jean Jautay, Jean-Paul Panié et Jean-Paul Perrier, a titre de stravail pratiques dans le cadre d'un programme post-troisieme cycle de l"Institut Auguste Comte, aujourd hui disparu. II nous a etté communiqué par la bibliotheqque centrale del'École Polytechnique (91128 Palaiseau Cedex - France).

(5) Yves Barel, Prospectlve ef analyse de syattems, Documentation française, Paris, 1971.

[6] Fabrice Roubelat, La prospective, mothodes et outils, dans le méme numero.

(7) Idem.

(B) Idem.

(9) Une approche plus aformalisten faisant appel aux methodes d'impacts croises, entierement infor matisbes, permettrait aujourd'hui d'obtenir une probabilisation plus objective des differents scénarios et par suite de les hierarchiser du plus ou moins probable. Elle corrige les opinions brutes exprimées par les experts en tenant compte des interdépendances entre les hypothèses sur les variables-clés de l'analyse ice que ne perrnet pas la méthode Delphi).

(10) Michel Godet, De ranticipation a raction: menuel de prospective et de etratígle, Dunod, 1991. Traduction anglaise: From Anticipation to Action, UNESCO, 1993, Voir aussil'article de Fabrice Roubelat dejà cité.

(11) Idem.

(12) Bernard Cazes et collaborateurs, Entrer dane lo XXI sibcle: essal sur I'avenir de ridentite française, Éditions La Découverte, Documentation française, 1990. 